\title{
A Rapid Bonding Method for Fabricating Mixing Microfluidic Chip Based on Micromachining Technology
}

\author{
Junyao Wang, ${ }^{1}$ Qi Hou, ${ }^{1}$ Huan Liu ${ }^{D},{ }^{1}$ Qi Sun, ${ }^{1}$ and Hengyi Yuan ${ }^{2}$ \\ ${ }^{1}$ School of Mechanical Engineering, Northeast Electric Power University, Jilin 132012, China \\ ${ }^{2}$ College of Mechanical Engineering, Jilin Engineering Normal University, Changchun 130052, China \\ Correspondence should be addressed to Huan Liu; 20192850@neepu.edu.cn
}

Received 11 July 2020; Revised 15 August 2020; Accepted 21 September 2020; Published 17 October 2020

Academic Editor: P. Davide Cozzoli

Copyright (C) 2020 Junyao Wang et al. This is an open access article distributed under the Creative Commons Attribution License, which permits unrestricted use, distribution, and reproduction in any medium, provided the original work is properly cited.

In this paper, a rapid bonding method is reported to fabricate a mixing microfluidic chip. The chip is composed of a glass upper plat and a polyurethane bottom plat. The upper plat with a microchannel is obtained through micromachining technology. And the bottom plat is manufactured via mixing the solution with the component of isocyanic acid and polyether polyol. The influence of the solution's proportion on the contact angle and bonding force is investigated to enhance the hydrophilicity and the bonding strength. Furthermore, a mixing experiment is implemented to verify the chip's bonding effect. The experimental results demonstrate that with increasing the proportions of isocyanic acid and polyether polyol, the contact angle and the bonding force increase at first and then decrease gradually. That is attributed to the variation of internal porous structure for different proportions. Considering the contact angle and the bonding force synthetically, the optimal proportion of $3.7 \mathrm{w} / \mathrm{w}$ is confirmed. The mixing efficiency is increased from 0.157 to 0.824 . Compared with other bonding methods, the method in this paper has the advantages of high efficiency and high bonding strength.

\section{Introduction}

Microfluidic chips [1] with microchannel, micropump, microvalve, and other units have been widely applied [2] to DNA analysis, immunoassay, and so on, owing to the main feature of rapidity, high efficiency, and low consumption [3, 4]. Among them, a mixing microfluidic chip [5] is utilized to realize the functions including rapid mixing and analysis. Remarkably, due to its excellent performance, the mixing microfluidic chip has applications in such fields as biological analysis and chemical detection [6]. Furthermore, in order to obtain the chips with different substrates, the technologies including channel fabricating [7] and chip bonding [8] are investigated.

Thereinto, the bonding [9] is a crucial processing step [10]. Currently, a variety of bonding methods [11] are employed to achieve the bonding of the different materials including glass/silicon, glass/glass, glass/PDMS, PDMS/PDMS, and PMMA/PMMA. To be noted, due to different properties of the material, the complexity for different bonding methods is not inconsistent $[12,13]$. For instance, an anodic bonding method is adopted to achieve the bonding of glass/silicon [14]. Nonetheless, an electrostatic bonding machine with the expensive value is essential. Remarkably, the simple bonding machine is used to implement the bonding of PMMA/PMMA [15], whereas the crack is more likely to happen owing to the lack of bonding strength. Dramatically, the bonding of glass/glass [16] compensates for the insufficiency of the former. A twostep bonding method including prebonding bonding and hot bonding is utilized to enhance the bonding strength. Regrettably, the low success rate of prebonding has become a bottleneck restricting its rapid development [17]. Fortunately, the plasmaassisted hot bonding is employed to improve the bonding success rate of glass/PDMS and PDMS/PDMS [18]. Nevertheless, the above methods require a bonding time [19] of at least half an hour. Accordingly, a rapid bonding method of the glass and polyurethane needs to be further investigated for fabricating mixing microfluidic chips.

In this paper, the bonding of glass/polyurethane is accomplished to manufacture mixing microfluidic chip through micromachining technology. Based on the chip, the influence of the solution's proportion on the contact 

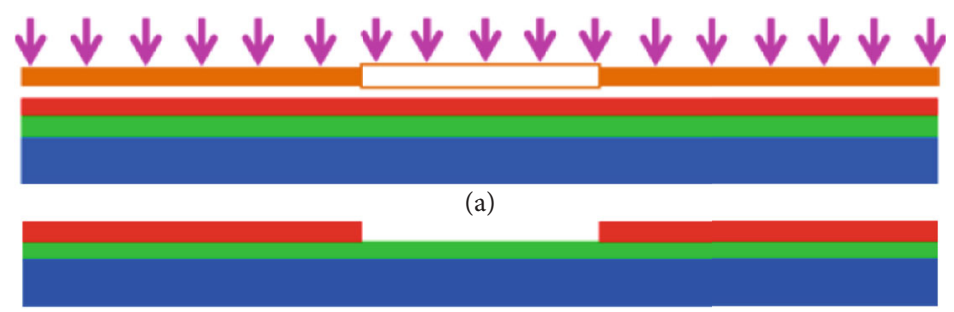

(b)

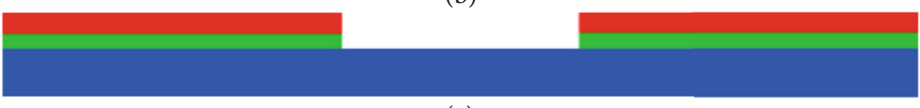

(c)

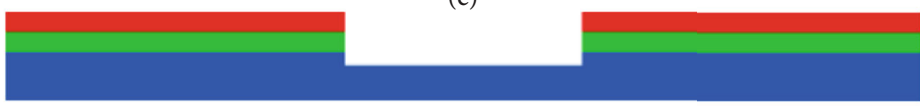

(d)

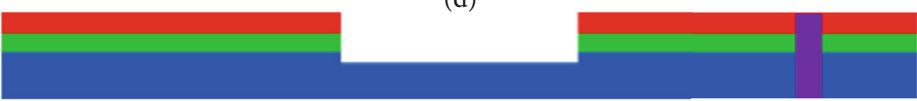

(e)

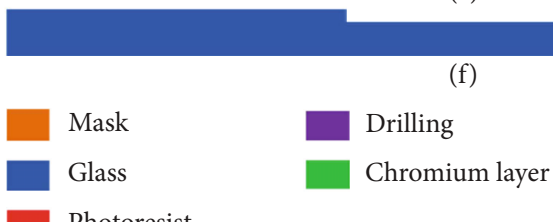

Figure 1: The manufacturing process of a glass microfluidic chip. (a) Photolithography. (b) development. (c) Chromium etching. (d) Glass etching. (e) Ultrasonic drilling. (f) Removing residual.

angle and bonding force is analyzed to enhance the hydrophilicity and the bonding strength of mixing microfluidic chip. At the end of the article, the mixing experiment of the deionized water and the blue ink is demonstrated to verify the bonding effect.

\section{Mixing Experiment Preparation}

The mixing microfluidic chip is composed of a glass upper plat and a polyurethane bottom plat. The glass plat with a layer of chromium is adopted to fabricate the upper plat. And the schematic illustration of the fabrication process is exhibited in Figure 1. The detailed steps are as follows: (1) exposure for about $60 \mathrm{~s}$, (2) development for about $10 \mathrm{~s}$, (3) wet etching for chromium, (4) wet etching for glass, (5) ultrasonic drilling, and (6removing surface residual. The bottom plat is established by mixing the isocyanic acid and the polyether polyol with certain proportions. And that is located in the oven with $40^{\circ} \mathrm{C}$ for $30 \mathrm{~min}$.

The bottom plat is manufactured via mixing the solution with the component of isocyanic acid and polyether polyol. And then, the upper plat and the bottom plat are confined together to form the mixing microfluidic chip, as shown in Figure 2. It can be seen that the microchannel with the width of $100 \mu \mathrm{m}$ and the depth of $30 \mu \mathrm{m}$ has an acute angle of $30^{\circ}$. In addition, the diameter of the three reservoirs is $2 \mathrm{~mm}$.

Based on the above mixing microfluidic chip, the deionized water and the blue ink are injected simultaneously into the microchannel of the chip utilizing the LSP02-2B Dual Channels Syringe Pump for the mixing experiment. The mixed images of different moments are taken for analyzing

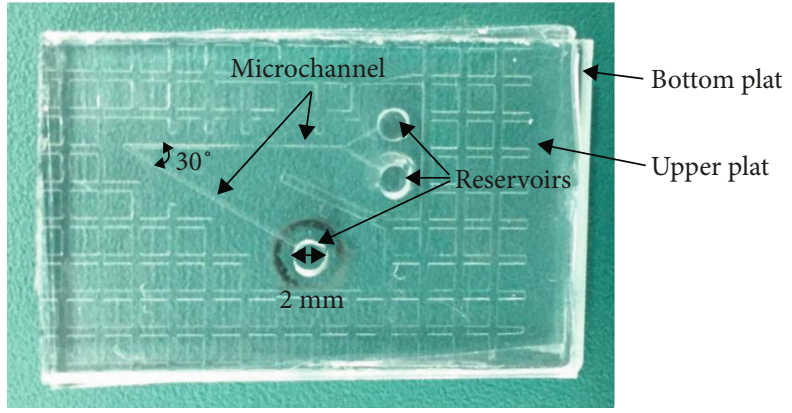

FIgURE 2: The mixing microfluidic chip. The microchannel with the width of $100 \mu \mathrm{m}$ and the depth of $30 \mu \mathrm{m}$ has an acute angle of $30^{\circ}$. In addition, the diameter of the three reservoirs is $2 \mathrm{~mm}$.

the mixing effect. In this section, the schematic diagram of the bonding and the injection process is shown in Figure 3.

\section{Analysis and Discussion}

3.1. Contact Angle. In order to estimate the performance of the mixing microfluidic chip (i.e., its hydrophilicity), the contact angle is selected as a measurement standard. And the effect of the proportions of isocyanic acid and polyether polyol on contact angle is investigated, as shown in Figure 4(a). It can be seen that the contact angle increases at first and then decreases gradually with increasing the proportions of isocyanic acid and polyether polyol. Specifically, the isocyanic acid and the polyether polyol are mixed with a certain percentage to form the polyurethane of porous structure [20,21]. Furthermore, the number of holes increases with increasing the 


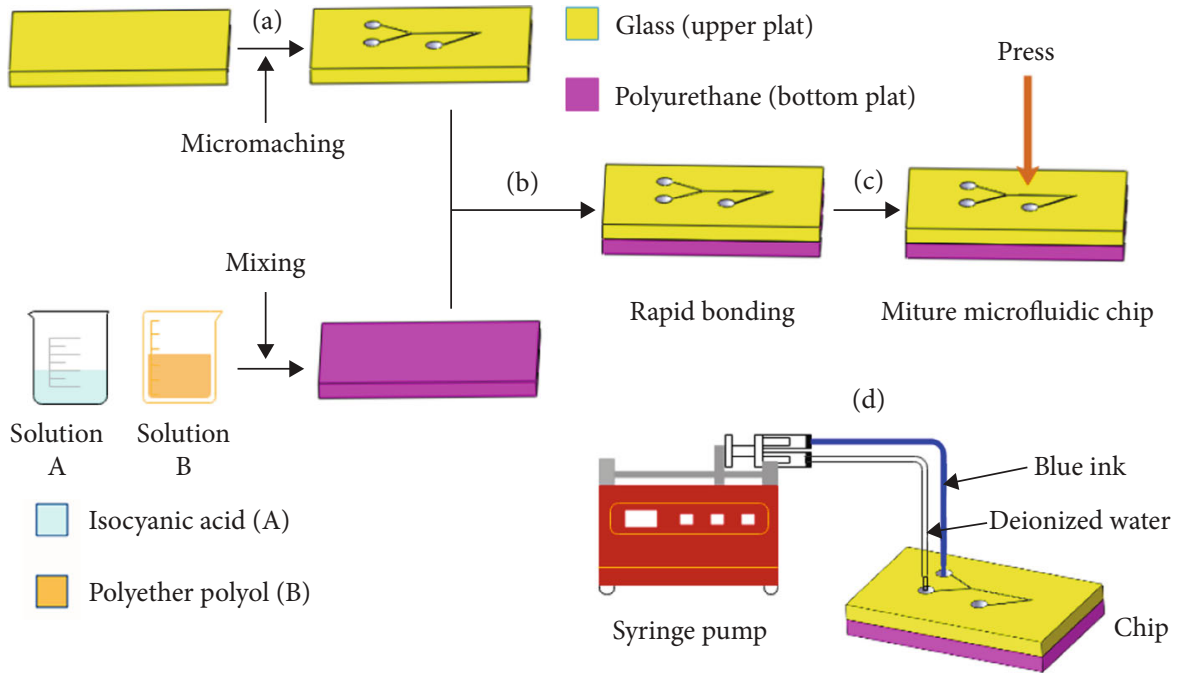

FIgURE 3: The process of bonding and injection. (a-c) A rapid bonding method is utilized to achieve the mixing chip of glass and polyurethane. (d) The deionized water and the blue ink are injected simultaneously into the microchannel of the chip for the mixing experiment.

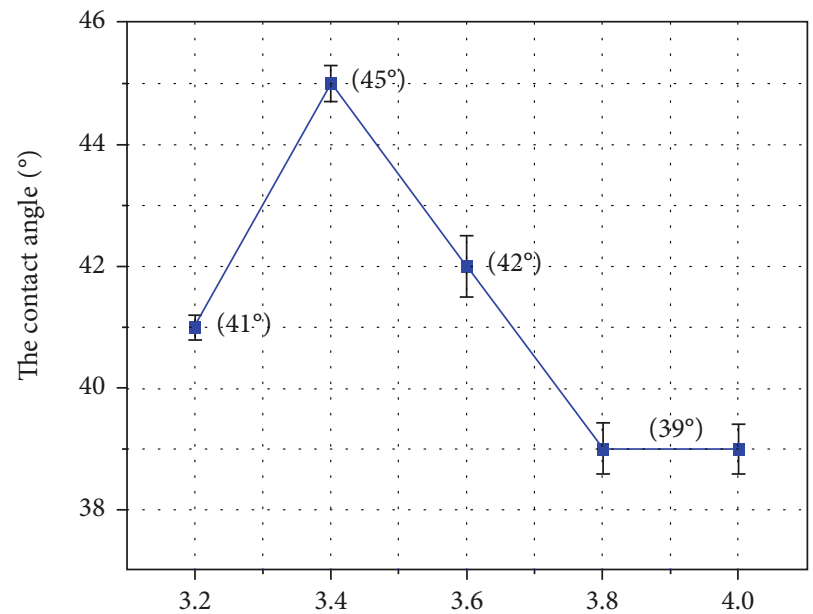

The proportions of isocyanic acid and polyether polyol (W/W)

(a)

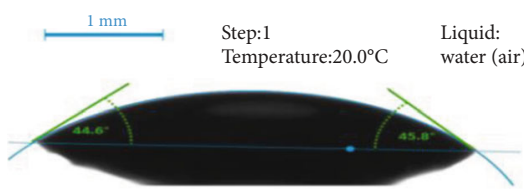

(c)

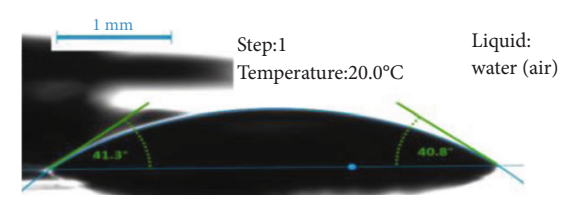

(b)

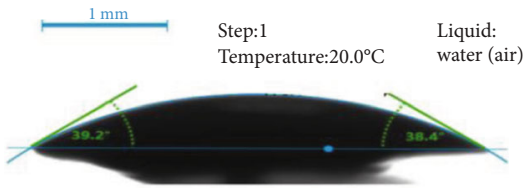

(d)

Figure 4: (a) The influence of the proportions of isocyanic acid and polyether polyol. (b-d) The photographs of contact angle with different proportions $(3.2 \mathrm{w} / \mathrm{w}, 3.4 \mathrm{w} / \mathrm{w}, 3.8 \mathrm{w} / \mathrm{w})$.

proportion of isocyanic acid. And that leads to a rougher surface and larger contact angle. Taking the proportion of $3.4 \mathrm{w} / \mathrm{w}$ as an example, the contact angle is the largest, but the hydrophilicity of the chip surface is the worst. Remarkably, when the proportions of isocyanic acid and polyether polyol is in the range of 3.8 to $4.0 \mathrm{w} / \mathrm{w}$, the contact angle turns out to be the smallest and the surface hydrophilicity is the best. A smaller contact angle will make it easier for the detected liquid passing into the microchannel. The photographs of contact angle with different proportions are exhibited in Figures 4(b)-4(d). And the schematic diagram of the relationship including the number of holes, surface roughness, contact angle, and hydrophilicity is shown in Figure 5.

3.2. Bonding Force. The bonding is a crucial processing step. Furthermore, the bonding quality depends mainly on the 

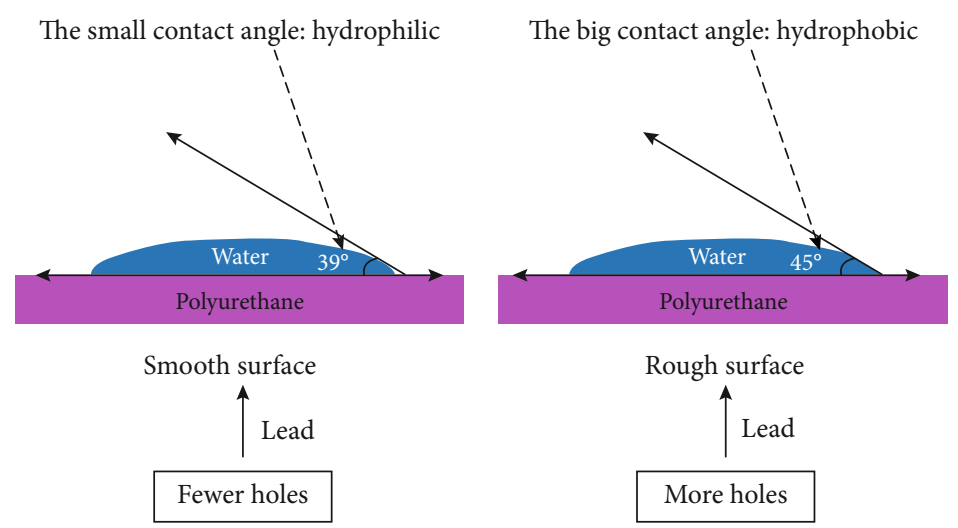

FIgure 5: The schematic diagram of the relationship among the number of holes, surface roughness, contact angle, and hydrophilicity.

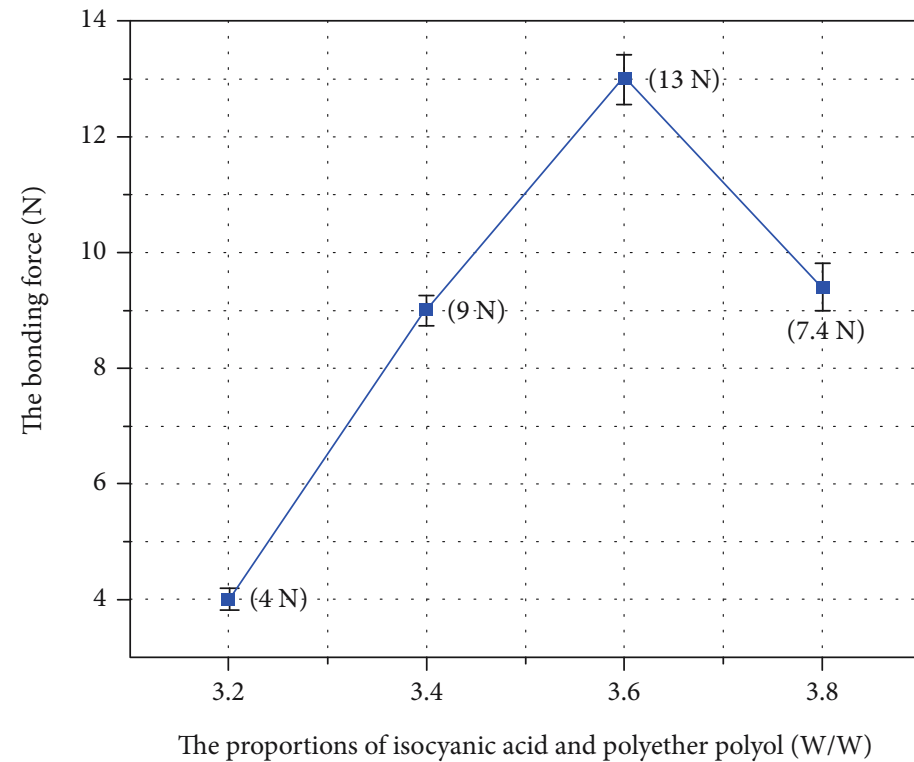

(a)

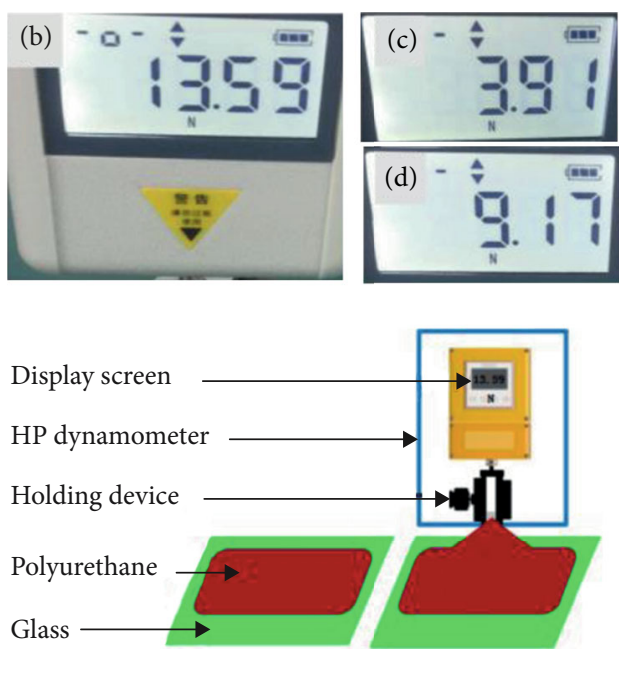

(e)

Figure 6: (a) The influence of the proportions of isocyanic acid and polyether polyol on the bonding force. (b)-(d) The photographs of the bonding force with different proportions. (e) A schematic diagram of the measuring method of the bonding force.

bonding force between the substrate and the cover. In other words, the higher the bonding force is, the better the bonding quality is, and the better the seal performance of the chip is. In order to estimate the performance of the mixing microfluidic chip (i.e., its bonding quality), the bonding force is selected as another measurement standard. And then, the effect of the proportions of isocyanic acid and polyether polyol on bonding force is investigated, as shown in Figure 6(a). To be noted, the bonding force increases firstly and then decreases with increasing the proportion of isocyanic acid and polyether polyol. The reason for this phenomenon is the same as that of the contact angle. It is worth mentioning that when the proportion of isocyanate and polyether polyols is $3.6 \mathrm{w} / \mathrm{w}$, the bonding force is the largest. In addition, the bonding forces with different proportions are shown in Figures 6(b)-6(d), and the measuring method of the bonding force is depicted in Figure 6(e). Considering the contact angle and the bonding force synthetically, the optimal proportion of $3.7 \mathrm{w} / \mathrm{w}$ is confirmed.

3.3. Mixing Efficiency. The deionized water and the blue ink are utilized to implement the mixing experiment. In order to investigate the mixing characteristics of the two solutions, equation (1) and equation (2) are introduced to evaluate the mixing effect. In equation (1), the concentration value $Y$ is obtained through bringing the gray value $X$. The gray values for $26 \mathrm{~s}, 30 \mathrm{~s}$, and $34 \mathrm{~s}$ are 241,203, and 188, respectively.

$$
Y=357.28661-2.50297 X+0.00437 X^{2}
$$

From equation (2), $I_{N}$ defined in [22] as the concentration mixing index is calculated to estimate the mixing 

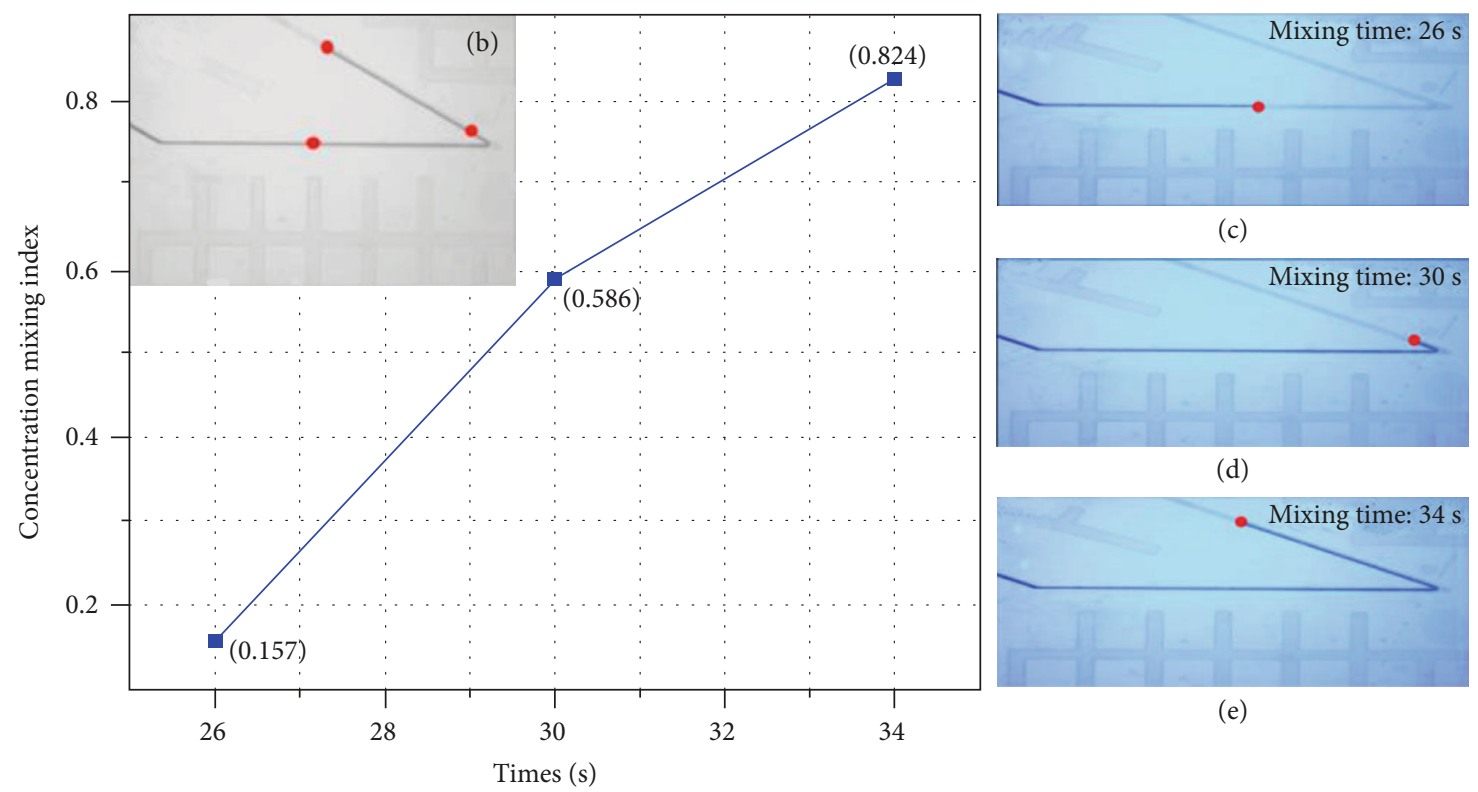

(c)

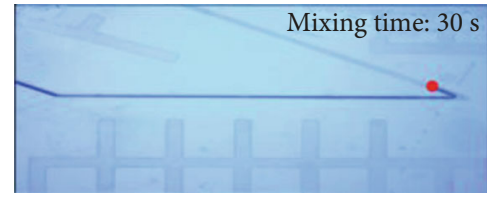

(d)

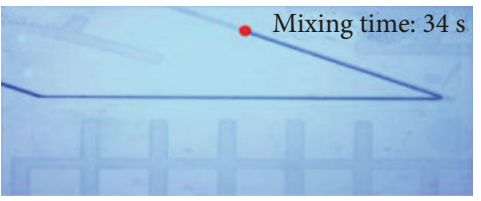

(e)

(a)

FIgURE 7: Demonstration of mixing efficiency. (a) The variation of concentration mixing index over time. (b) The gray image of the mixed solution including three gray values at $26 \mathrm{~s}, 30 \mathrm{~s}$, and $34 \mathrm{~s}$. (c)-(e) Three mixed pictures pictured at $26 \mathrm{~s}, 30 \mathrm{~s}$, and $34 \mathrm{~s}$, respectively.

TABLE 1: Comparison of different bonding methods.

\begin{tabular}{lccc}
\hline Object & Equipment & Bonding step & Bonding time \\
\hline Glass/glass & Muffle furnace & Two & More than 12 hours \\
Glass/silicon & Electrostatic bonding machine & One & Several hours \\
Glass/PDMS and PDMS/PDMS & Plasma cleaning machine & Two & More than half an hour \\
PMMA/PMMA & Hot-embossing machine & One & More than half an hour \\
Glass/polyurethane & No & Two & Only 2 minutes \\
\hline
\end{tabular}

efficiency through bringing the concentration value $Y$. In general, the higher the mixing index is, the better the mixing efficiency is.

$$
I_{N}=1-\frac{1}{50 \%}(Y-50 \%) .
$$

The relationship between the mixing index and the time is demonstrated in Figure 7(a). It can be seen that the mixing index increases over time. For instance, the corresponding mixing index at $26 \mathrm{~s}, 30 \mathrm{~s}$, and $34 \mathrm{~s}$ are $0.157,0.586$, and 0.824 , respectively. To be noted, when the time is more than $30 \mathrm{~s}$, the growth rate of the mixing index is higher than that $30 \mathrm{~s}$ ago. That is attributed to the promoting effect of the acute angle channel (i.e., $30^{\circ}$ ). The mixing efficiency is also related to the channel structure. In addition, the mixing pictures at $26 \mathrm{~s}, 30 \mathrm{~s}$, and $34 \mathrm{~s}$ are shown, respectively, in Figures $7(\mathrm{c})-7(\mathrm{e})$. Among them, three gray values corresponding to the three points are exhibited in Figure 7(b).

3.4. Comparison of Bonding Methods of Different Materials. Some bonding methods of different materials are listed in
Table 1. It is worth noting that from the point of view of the bonding device, except for the method described in this article, other methods require specific devices to achieve bonding [23-25]. As for the bonding step, glass/glass [16], glass/PDMS, and PDMS/PDMS [18] require two steps for the bonding, while glass/silicon [14], PMMA/PMMA [15], and glass/polyurethane require only one step. From the perspective of bonding time [19], for all materials except glass/polyurethane, it takes 30 minutes or more. To be noted, for glass/polyurethane, it only takes 2 minutes. Through a comprehensive comparison of the above three aspects, the glass/polyurethane rapid bonding method avoids the problems of equipment utilizing and long bonding time. In summary, the proposed bonding method in this paper has the advantages of direct bonding, simple bonding step, and short bonding time.

\section{Concluding Remarks}

In order to manufacture mixing microfluidic chip, a rapid bonding method based on a micromachining technology is proposed. The influences of the proportions of the mixed 
solution on contact angle and bonding force are investigated to obtain the optimal proportion. Furthermore, a mixing microfluidic chip is fabricated based on the optimal proportion of mixed solution. The results of the mixing experiment demonstrate that with increasing the proportions of isocyanic acid and polyether polyol, the contact angle and the bonding force increase at first and then decrease gradually. Considering the relevant parameters including contact angle and bonding force comprehensively, the optimal proportion turns out to be $3.7 \mathrm{w} / \mathrm{w}$. The mixing experiment results show that the mixing efficiency is increased from 0.157 to 0.824 over time. In summary, a rapid bonding method of mixing microfluidic chip has the characteristics of high efficiency and high bonding strength.

\section{Data Availability}

The (data type) data used to support the findings of this study are included in the article. Some of the important data supporting the conclusions of the study are shown in Figures 4, 6 , and 7.

\section{Conflicts of Interest}

The authors declare no conflicts of interest.

\section{Acknowledgments}

This project is supported by the National Natural Science Foundation of China (Grant No. 51505077), Project Agreement for Science and Technology Development of Jilin Province (JJKH20200105KJ), and Science and Technology Innovation Development Project of Jilin City (201750230, 20166013, 20166012).

\section{References}

[1] H. Jiang, X. F. Lv, and K. X. Zhao, "Progress of aptamer screening techniques based on microfluidic chips," Chinese Journal of Analytical Chemistry, vol. 48, no. 5, pp. 590-600, 2020.

[2] J. Wang, G. Wang, M. Chen et al., "An integrated microfluidic chip for treatment and detection of microalgae cells," Algal Research, vol. 42, article 101593, 2019.

[3] S.-L. Hong, M. Tang, Z. Chen et al., "High-performance multiplex microvalves fabrication and using for tumor cells staining on a microfluidic chip," Biomedical Microdevices, vol. 21, no. 4, 2019.

[4] J.-S. Liu, Y.-Y. Zhang, Z. Wang et al., "Design and validation of a microfluidic chip with micropillar arrays for threedimensional cell culture," Chinese Journal of Analytical Chemistry, vol. 45, no. 8, pp. 1109-1114, 2017.

[5] A. Mariotti, C. Galletti, R. Mauri, M. V. Salvetti, and E. Brunazzi, "Steady and unsteady regimes in a T-shaped micro-mixer: synergic experimental and numerical investigation," Chemical Engineering Journal, vol. 341, pp. 414-431, 2018.

[6] N. R. Kuppusamy, N. N. N. Ghazali, R. Saidur, and M. E. Niza, "Optimum design of triangular shaped micro mixer in micro channel heat sink," International Journal of Heat and Mass Transfer, vol. 91, pp. 52-62, 2015.
[7] C. Sudarsan, K. Sajun Prasad, S. Hazra, and S. K. Panda, "Forming of serpentine micro-channels on SS304 and AA1050 ultrathin metallic sheets using stamping technology," Journal of Manufacturing Processes, vol. 56, pp. 1099-1113, 2020.

[8] C. Chen, S. Noh, H. Zhang et al., "Bonding technology based on solid porous Ag for large area chips," Scripta Materialia, vol. 146, pp. 123-127, 2018.

[9] P. C. Chen and L. H. Duong, "Novel solvent bonding method for thermoplastic microfluidic chips," Sensors and Actuators B: Chemical, vol. 237, pp. 556-562, 2016.

[10] Y. Q. Fan, H. L. Wang, and Y. J. Zhang, "Review on low-cost fabrication technology for polymer microfluidic chip," Transducer and Microsystem Technologies, vol. 38, pp. 1-5, 2019.

[11] H. D. Lynh and C. Pin-Chuan, "Novel solvent bonding method for creation of a three-dimensional, non-planar, hybrid PLA/PMMA microfluidic chip," Sensors and Actuators A: Physical, vol. 280, pp. 350-358, 2018.

[12] R. Kodzius, K. Xiao, J. Wu et al., "Inhibitory effect of common microfluidic materials on PCR outcome," Sensors and Actuators B-Chemical, vol. 161, no. 1, pp. 349-358, 2012.

[13] Y. N. Jo, P. Santhoshkumar, K. Prasanna, K. Vediappan, and C. W. Lee, "Improving self-discharge and anti-corrosion performance of $\mathrm{Zn}$-air batteries using conductive polymercoated Zn active materials," Journal of Industrial and Engineering Chemistry, vol. 76, pp. 396-402, 2019.

[14] J. Xu, C. Wang, T. Wang, Y. Liu, and Y. Tian, "Direct bonding of silicon and quartz glass using VUV/O3 activation and a multistep low-temperature annealing process," Applied Surface Science, vol. 453, pp. 416-422, 2018.

[15] C. H. Li, H. Wang, S. X. Wang, and J. G. Cui, "Research on bonding technology based on PMMA material," China Plastics Industry, vol. 46, pp. 117-122, 2018.

[16] C. Wang, H. Fang, S. Zhou et al., "Recycled low-temperature direct bonding of Si/glass and glass/glass chips for detachable micro/nanofluidic devices," Journal of Materials Science \& Technology, vol. 46, pp. 156-167, 2020.

[17] Y. Li, R. Tian, Y. Xiao et al., "Improved the quality of the glass/glass laser bonding through the optimization of glass powder size in planetary ball mill," Molecular Crystals and Liquid Crystals, vol. 651, no. 1, pp. 273-281, 2017.

[18] V. Bagiatis, G. W. Critchlow, D. Price, and S. Wang, "The effect of atmospheric pressure plasma treatment (APPT) on the adhesive bonding of poly(methyl methacrylate) (PMMA)-to-glass using a polydimethylsiloxane (PDMS)based adhesive," International Journal of Adhesion and Adhesives, vol. 95, article 102405, 2019.

[19] A. Doroudi, A. Shamsipur, H. Omidvar, and M. Vatanara, "Effect of transient liquid phase bonding time on the microstructure, isothermal solidification completion and the mechanical properties during bonding of Inconel 625 superalloy using Cr-Si-B-Ni filler metal," Journal of Manufacturing Processes, vol. 38, pp. 235-243, 2019.

[20] A. V. Menon, G. Madras, and S. Bose, "Shape memory polyurethane nanocomposites with porous architectures for enhanced microwave shielding," Chemical Engineering Journal, vol. 352, pp. 590-600, 2018.

[21] X. Dang, Y. Li, and M. Yang, "Biodegradable waterborne polyurethane grafted with gelatin hydrolysate via solvent-free copolymerization for potential porous scaffold material," Journal of the Mechanical Behavior of Biomedical Materials, vol. 92, pp. 79-89, 2019. 
[22] X. L. Wang, R. Y. Zhang, G. S. Chen, and S. D. Li, "Concentration distribution evaluation technique for T-shaped micromixer," Chinese Journal of Analytical Chemistry, vol. 9, pp. 1241-1244, 2008.

[23] S. R. Mahmoodi, P. K. Sun, M. Mayer, and R. S. Besser, "Gasassisted thermal bonding of thermoplastics for the fabrication of microfluidic devices," Microsystem Technologies, vol. 25, no. 10, pp. 3923-3932, 2019.

[24] J. Xu, C. Wang, Y. Tian, B. Wu, S. Wang, and H. Zhang, "Glass-on-LiNbO3 heterostructure formed via a two-step plasma activated low-temperature direct bonding method," Applied Surface Science, vol. 459, pp. 621-629, 2018.

[25] Z. Yin, "Rapid prototyping of PET microfluidic chips by laser ablation and water-soaking bonding method," Micro \& Nano Letters, vol. 13, no. 9, pp. 1302-1305, 2018. 